Т.А. МАКАРЕНКО, Д.М.Н., Д.Е. НИКИФОРОВА, И.О. УЛЬЯНОВА, К.М.Н.

Красноярский государственный медицинский университет им. проф. В.Ф. Войно-Ясенецкого Минздрава России

\title{
ВНУТРИМАТОЧНЫЕ СИНЕХИИ:
}



\begin{abstract}
Статья посвящена проблеме хирургического лечения и профилактики рецидивов внутриматочных синехий (ВС) и крайней их формы - синдрома Ашермана. Актуальность ВС характеризуется высокой частотой бесплодия, привычного невынашивания, аменореи и гипоменструального синдрома. В статье описаны принципы ведения больных с ВС, особенности их хирургического лечения и профилактики рецидивов заболевания. Приведены собственные клинические данные, обобщающие опыт лечения 64 женщин с ВС. Всем больным был выполнен гистероскопический адгезиолизис. В послеоперационном периоде 48 женщинам (I группа) в полость матки вводился противоспаечный барьер на основе гиалуроновой кислоты и карбоксиметилцеллюлозы - Антиадгезин. Группу контроля (II группа) составили 16 женщин, которым Антиадгезин не вводили. В результате проведенного исследования было выявлено, что среди пациенток I группы констатированы значимо меньшее количество рецидивов ВС, а также достоверно большее число случаев наступившей беременности. Таким образом, для комплексного лечения пациенток с ВС решающее значение имеют не только бережное и полное рассечение спаек, но и активный послеоперационный реабилитационный период, включающий профилактику рецидива адгезиогенеза при помощи внутриматочного введения противоспаечных барьеров.
\end{abstract}

Ключевые слова: внутриматочные синехии, синдром Ашермана, гистероскопия, адгезиолизис, рассечение спаек, противоспаечный барьер, Антиадгезин.

T.A. MAKARENKO, MD, D.E. NIKIFOROVA, I.O. ULYANOVA, PhD in medicine, Voino-Yasenetsky Krasnoyarsk State Medical University of the Ministry of Health of Russia

INTRAUTERINE SINECHIA: FEATURES OF SURGICAL TREATMENT AND PREVENTION OF RECURRENCE

The article is devoted to the issues of surgical treatment and prevention of recurrence of intrauterine sinechia (IUS) and its severe condition Asherman syndrome. The topicality of the IUS is characterized by a high incidence of infertility, common miscarriage, amenorrhea and hypomenstrual syndrome. The article describes the principles for management of patients with IUS, the features of surgical treatment and the prevention of recurrence of the disease. The authors present their own clinical data summarizing the experience in treating 64 women with IUS. All patients underwent a hysteroscopic adhesiolysis. In the postoperative period, 48 women (group 1) received an adhesion barrier based on hyaluronic acid and carboxymethylcellulose Antiadhesin injected into the cavity of the uterus. 16 women (control group 2) did not receive Antiadgesin. The study showed that significantly fewer relapses and a significant increase in commenced pregnancy were detected among patients in group 1. As can be seen from the above, not only the careful and complete dissection of the adhesions, but also the active postoperative rehabilitation period, which includes the prevention of relapsing adhesiogenesis using intrauterine injections of adhesion barriers, is crucial for the comprehensive treatment of patients with IUS.

Keywords: intrauterine sinechia, Asherman syndrome, hysteroscopy, adhesiolysis, adhesiotomy, adhesion barrier, Antiadhesin.

нутриматочные синехии (BC) (греч. sinechiae слипание) - спайки, ведущие к частичной или полной облитерации полости матки и/или цервикального канала соединительной тканью.

Впервые ВС, возникшие после выскабливания полости матки, были описаны в конце XIX в. Heinrich Fritsch, но их клиническую значимость доказал Asherman в 1948 г. на примере пациентки с вторичной аменореей после травматичных родов [1]. В настоящее время термин «синдром Ашермана» является собирательным понятием и часто используется в случаях диагностики любых видов ВС. Несмотря на то, что о ВС известно больше века, эта проблема до сих пор остается нерешенной, в связи с чем продолжается разработка способов профилактики, диагностики и лечения данной патологии.

Актуальность ВС связана с высокой частотой бесплодия, привычного невынашивания, аменореи и гипоменструаль- ного синдрома при данной патологии [2]. Так, доля ВС у пациенток с нарушениями менструального цикла и репродуктивными потерями в анамнезе составляет от 2,8\% до 45,5\% [3]. В связи с развитием инновационных методов обработки изображения при гистероскопии, которая является «золотым стандартом» диагностики ВС, частота этого заболевания неуклонно растет и достигает 22\% среди бесплодных пар [4-6]. Кроме того, в последнее время с ВС связывают нарушение процессов имплантации в программах вспомогательных репродуктивных технологий (ВРТ), рост числа преждевременных родов, преждевременный разрыв плодных оболочек, аномальную локализацию плаценты и неправильное положение плода [7].

На сегодняшний день формирование ВС наиболее часто связывают с повреждением базального слоя эндометрия, наступившим в результате различных внутриматочных вмешательств [8]. Наиболее частой причиной развития 
адгезивного процесса в полости матки являются выскабливания ее слизистой по поводу прервавшейся или неразвивающейся беременности, а также осложнений послеродового периода. Однако в связи с появлением новых внутриматочных операций, выполняемых по поводу субмукозных миоматозных узлов, полипов, аномалий развития матки и т. д., в настоящее время в клинической практике стоит выделять и эту группу пациенток, предрасположенных к формированию ВC [9-11]. Наложение компрессионных швов при послеродовом кровотечении в последующем также способствует развитию ВС [12].

Ряд авторов утверждают, что критический период, в течение которого появляются спайки, - от 3 до 5 дней после операции [13]. Этот процесс усиливается рядом факторов, которые нарушают физиологический фибринолиз: ишемия, посттравматическое воспаление, наличие крови, инородные тела [14]. Спайки могут вовлекать различные слои как эндометрия, так и миометрия. Во многих случаях передняя и задняя стенки матки адгезируются между собой, в других случаях спайки образуются только локально. Количество спаек определяет степень тяжести процесса: легкая, умеренная или тяжелая. ВС могут быть тонкими или плотными, фиброзированными.

В настоящее время «золотым стандартом» диагностики и лечения ВС является гистероскопия. При гистероскопии синехии определяются как белесоватые бессосудистые тяжи - сращения между стенками матки различной плотности и протяженности, нередко уменьшающие объем полости матки, а иногда полностью ее облитерирующие. Синехии могут располагаться также в цервикальном канале, вызывая его заращение и препятствуя доступу в полость матки и оттоку содержимого из нее. Нежные синехии выглядят как тяжи бледно-розового цвета в виде паутины, иногда видны проходящие в них сосуды. Более плотные сращения определяются как плотные белесоватые тяжи, располагающиеся, как правило, по боковым стенкам, реже - по центру полости матки. Множественные поперечные синехии обусловливают частичное заращение полости матки с множеством полостей различной величины в виде углублений (отверстий). Иногда эти отверстия ошибочно принимают за устья маточных труб [15].

Ведение пациенток с ВС включает несколько этапов:

1. Хирургическое лечение (адгезиолизис).

2. Профилактика рецидивов (использование внутриматочных устройств, противоспаечных барьеров).

3. Восстановление эндометрия (циклическая гормонотерапия, физиолечение, внутриматочное введение кавитированных растворов и т. д.) в течение 2-3 менструальных циклов.

4. Послеоперационная оценка состояния полости матки (через 2-3 мес. после разрушения синехий по окончании проведения реабилитационных мероприятий): УЗИ, гистеросальпингография, контрольная офисная гистероскопия.

Первым этапом лечения ВС является проведение операционной гистероскопии с целью рассечения спаек под визуальным контролем. Разделение синехий в зависимости от их плотности производится тубусом гистероскопа, эндоскопическими ножницами, щипцами (5 Шр), гистерорезектоскопом, лазером. Однако наиболее благоприятным способом разделения спаек считается механический, т. к. использование высоких энергий связано с потенциальным риском термического повреждения интактного эндометрия [16]. Категорически не рекомендуется использование слепого кюретажа с целью разрушения ВС в связи с высоким риском дополнительного травмирования уже поврежденного эндометрия и развития рецидива спаечного процесса.

При выполнении гистероскопии у пациенток с ВС можно выделить ряд технических особенностей:

1. Проводя гистероскопию при подозрении на ВC, не следует предварительно зондировать полость матки и расширять цервикальный канал. Выполнять гистероскопию целесообразно по технологии «no-touch» по S. Betocchi при помощи тонкого офисного гистероскопа с операционным каналом для хирургических инструментов.

2. Гистероскоп вводят в цервикальный канал под контролем зрения с постоянной подачей жидкости под давлением для расширения полости матки.

3. Если в цервикальном канале определяются синехии, их постепенно разрушают при помощи гидравлической препаровки, ножниц или щипцов.

4. В дальнейшем при диагностической гистероскопии определяют вид и протяженность синехий, степень заращения полости матки, обследуют область устьев маточных труб. При помощи гистероскопических микроинструментов постепенно проводят разрушение ВС. В случае тяжелых фиброзированных сращений офисный гистероскоп меняют на гистерорезектоскоп (после предварительного расширения цервикального канала) и проводят адгезиолизис при помощи электрической энергии.

5. При тяжелой степени ВС операцию целесообразно выполнять под контролем УЗИ и/или лапароскопии.

Очень важно, чтобы во время адгезиолизиса была восстановлена полость матки в своих нормальных размерах и сохранены островки эндометрия, за счет которых происходит его регенерация. Использование хирургической офисной гистероскопии с микроинструментами, а в случае необходимости - биполярной электроэнергии гистерорезектоскопа в соответствии с концепцией «See and treat» $[17,18]$, при условии дополнительного ультразвукового или лапароскопического контроля в сложных клинических случаях позволяет выполнять диагностику и бережное лечение $B C$.

Однако даже после самого грамотного (бережного и тщательного) разрушения ВС риск рецидивов остается высоким. В среднем частота рецидивирования легких и умеренных синехий в совокупности составляет около 30\%, тяжелых - в 2 раза больше (62,5\%) [19]. Так как рецидив ВС наступает в раннем послеоперационном периоде, наиболее важное значение имеет профилактика этого заболевания именно в течение первых дней после операции.

В литературе имеются указания на высокую эффективность различных методик профилактики рецидивов адгезивного процесса в полости матки. Среди них: использование различных внутриматочных устройств, катетера Foley, 
а также введение в полость матки разнообразных лекарственных препаратов и противоспаечных барьеров $[20,21]$.

Некоторые авторы для профилактики рецидива ВС предлагают введение в полость матки гелеобразных наполнителей (противоспаечных барьеров), препятствующих контакту травмированных стенок матки и образованию спаек. В систематическом обзоре A. Di Spiezio Sardo et al. представлено, что гелевые барьеры оказывают существенное клиническое влияние на профилактику ВС за счет высокого адгезионного эффекта и длительного пребывания на поврежденной поверхности [22].

В последние десятилетия в гинекологии в качестве профилактики интраперитонеальных и внутриматочных спаек стали широко применяться гиалуроновая кислота (ГК) и ее производные. ГК является одним из самых распространенных компонентов в ткани человека и участвует во многих биологических функциях, таких как механическая поддержка, клеточная миграция и пролиферация [23]. Исследованиями многих авторов доказана эффективность использования ГК в качестве профилактики внутриматочных адгезий в основной группе по сравнению с контрольной, где не использовался ни один из методов противорецидивной терапии [24]. Механизм действия ГК реализуется на очень ранней стадии спайкообразования (первые 3-4 дня) путем подавления адгезии фибробластов и тромбоцитов, активности макрофагов, а также путем ингибирования образования фибрина и создания защитного барьера на поврежденном участке ткани. Период полураспада ГК в организме - около 1-3 дней, она полностью расщепляется в организме ферментом гиалуронидазой в течение 4 сут.

В рекомендациях Королевского колледжа акушерства и гинекологии по применению противоспаечных средств в акушерстве и гинекологии (RCOG, Великобритания, 2013) указано, что все виды хирургических операций на органах брюшной полости и малого таза приводят к спаечному процессу. Это является основанием для использования противоспаечных средств во время хирургических вмешательств, в т. ч. внутриматочных. При этом наиболее эффективными противоспаечными средствами в акушерстве и гинекологии признаны производные ГК [25]. Американская ассоциация лапароскопических хирурговгинекологов (AAGL, 2013) рекомендует после проведения любых внутриматочных вмешательств применять барьер- ные противоспаечные средства (гели), в состав которых входит ГК [26].

Многие современные противоспаечные гелевые барьеры созданы на основе другого антиадгезивного компонента - карбоксиметилцеллюлозы, которая представляет собой медленно рассасывающийся простой эфир целлюлозы и гликолевой кислоты с высокой молекулярной массой (350 000 Да), который хорошо растворяется в воде, образуя гель, способный отделять серозные поверхности в течение периода регенерации тканей, действует как синтетический механический барьер. Механизм действия реализуется еще и за счет уменьшения активности фибробластов и предотвращения депонирования фибрина на поврежденной серозной поверхности, также замедляется движение клеток, направляющихся в очаг воспаления. В хирургии служит в качестве субстрата для закрепления и пролонгирования действия ГК на поверхности ткани. Элиминируется путем постепенного лизиса и поглощения фрагментов макрофагами.

Комбинация высокоочищенной натриевой соли ГК и карбоксиметилцеллюлозы в виде геля Антиадгезин ${ }^{\circledR}$ («Геньюэл Ко Лтд», Корея) предназначена для профилактики спайкообразования после любых операций на органах и тканях, где имеется такой риск, в т. ч. после внутриматочных вмешательств [27]. Терапевтический эффект геля Антиадгезин ${ }^{\circledR}$ связан с созданием искусственного временного барьера между поврежденными тканями, что обеспечивает эффективное разделение поверхностей на время их заживления. После аппликации геля в области операционного поля он прилипает к анатомическим поверхностям, не растекаясь, и образует вязкое смазывающее покрытие, которое обеспечивает скольжение соседних поврежденных поверхностей и предупреждает их слипание. Антиадгезин ${ }^{\circledR}$ является биодеградируемым покрытием, которое разделяет соприкасающиеся поверхности только на период критической фазы раневого заживления и послеоперационного спайкообразования, продолжающийся в течение 7 дней, не влияя при этом на нормально протекающие процессы регенерации. После применения гель полностью рассасывается. Данный противоспаечный гель обладает рядом важных характеристик: он прост в использовании, безопасен и инертен (не является очагом инфекции, фиброза, ангиогенеза и пр.), снабжен очень удобным для внутриматочного введения тонким аппликатором.

\section{Таблица 1. Жалобы больных обследуемых групп с ВС}

\begin{tabular}{|l|c|c|c|}
\hline Данные анамнеза и жалобы & $\begin{array}{c}\text { I группа обследуемых } \\
(\mathrm{n}=48) \text { Абс., \% }\end{array}$ & $\begin{array}{c}\text { II группа обследуемых } \\
(\mathrm{n}=16) \text { Абс., \% }\end{array}$ & $\begin{array}{c}\text { Достоверность } \\
\text { различий, } \mathrm{p}\end{array}$ \\
\hline Вторичная аменорея & $4(8,3 \%)$ & $2(12,5 \%)$ & $\mathrm{p}>0,05$ \\
\hline Гипоменструальный синдром & $22(45,8 \%)$ & $7(43,8 \%)$ & $\mathrm{p}>0,05$ \\
\hline Дисменорея & $11(22,9 \%)$ & $2(12,5 \%)$ & $\mathrm{p}>0,05$ \\
\hline Вторичное бесплодие & $34(70,8 \%)$ & $11(68,8 \%)$ & $\mathrm{p}>0,05$ \\
\hline Невынашивание беременности & $8(16,7 \%)$ & $3(18,8 \%)$ & $\mathrm{p}>0,05$ \\
\hline
\end{tabular}


Таблица 2. Данные анамнеза у больных обследуемых групп с ВС

\begin{tabular}{|c|c|c|c|}
\hline Данные анамнеза и жалобы & $\begin{array}{l}\text { I группа обследуемых } \\
\text { (n = 48) Абс., \% }\end{array}$ & $\begin{array}{l}\text { لІ группа обследуемых } \\
(n=16) \text { Абс., \% }\end{array}$ & $\begin{array}{l}\text { Достоверность } \\
\text { различий, p }\end{array}$ \\
\hline Выскабливание полости матки & $48(100 \%)$ & $16(100 \%)$ & $p>0,05$ \\
\hline Неудачные попытки ЭКО & $20(41,7 \%)$ & $6(37,5 \%)$ & $p>0,05$ \\
\hline Гистероскопия, адгезиолизис & $11(22,9 \%)$ & $4(25 \%)$ & $p>0,05$ \\
\hline
\end{tabular}

Материал и методы. Нами был проведен анализ результатов лечения 64 больных с ВС, прооперированных по поводу данной внутриматочной патологии. Больные были ретроспективно разделены на две клинические группы. Группу । составили 48 женщин, которым в послеоперационном периоде с целью профилактики рецидива развития ВС в полость матки вводился противоспаечный барьер Антиадгезин ${ }^{\circledR}$ (за период с 2016 по февраль 2018 г.). В группу II были включены 16 больных, которым противоспаечный барьер не вводили (период наблюдения с 2014 по 2016 г.). По характеру жалоб и особенностям анамнеза среди женщин I и II групп достоверных различий выявлено не было (табл. 1, 2).

Таким образом, больные с ВС наиболее часто обращались с жалобами на гипоменструальный синдром и вторичное бесплодие.

Как видно из данных, представленных в таблице 2, у всех больных с ВС в анамнезе имели место выскабливания слизистой полости матки по поводу различной патологии (остатки частей последа в послеродовом периоде, прерывание беременности, в т. ч. неразвивающейся), а у каждой пятой в анамнезе уже проводились гистероскопия или гистерорезектоскопия с рассечением ВС и неоднократные курсы консервативной терапии с целью реабилитации эндометрия.

Выраженность патологического процесса в полости матки определялась нами во время проведения офисной гистероскопии согласно классификации Европейской ассоциации гинекологов-эндоскопистов (ESH, 1989). При этом использовался офисный хирургический гистероскоп B.I.O.H. (Karl Storz). По степени тяжести ВС выделенные нами группы больных были сопоставимыми.

Во время гистероскопии всем пациенткам произведен адгезиолизис преимущественно при помощи гистероскопических ножниц (5Шр), введенных в операционный канал офисного гистероскопа B.I.O.H. (Karl Storz). Только у 6 женщин (5 больных I группы и 1 - II группы) дополнительно произведена резекция ВС при помощи биполярного гистерорезектоскопа (Karl Storz) в связи с наличием очень плотных фиброзированных синехий. у 3 больных ввиду выраженного спаечного процесса в полости матки был применен лапароскопический контроль, у 2 - ультразвуковой. Сразу по окончании операции всем пациенткам I группы внутриматочно вводился противоспаечный гель Антиадгезин ${ }^{\circledR}$. В связи с выраженной степенью адгезивного процесса в полости матки 25 (52,1\%) больным I группы в послеоперационном периоде было продолжено введение данного противоспаеч- ного барьера амбулаторно еще 1-3 раза с интервалом в 3-5 дней (табл. 3).

Результаты исследования. Через 2 мес. после рассечения ВС всем больным была выполнена контрольная офисная гистероскопия. Уже на догоспитальном этапе было выяснено, что восстановление менструальной функции наблюдалось у большинства больных. У 3 пациенток (2 - из I группы и 1 - из II группы), имевших до операции вторичную аменорею, в послеоперационном периоде отмечены регулярные, но скудные менструалоподобные реакции. Дисменорея сохранялась лишь у 2 женщин II группы.

У всех больных I группы во время контрольной гистероскопии визуализировалась полость матки достаточного объема. Наличие рецидива ВС было диагностировано лишь у 4 (8,3\%) пациенток I группы: у 1 женщины с исходно III степенью заболевания, у 2 - с исходно IV степенью и у 1 - с Vа степенью. При этом во всех случаях рецидива спайки были единичными (І степени по классификации ESH, 1989) и легко разрушались тубусом гистероскопа. Во II группе рецидив ВС имел место у 5 (31,3\%) больных (p = 0,038): у 2 (12,5\%) больных с исходно IV степенью заболевания и у 3 (18,8\%) - с исходно III степенью, при этом у 1 больной визуализировалась «туннелированная» матка, что является крайне неблагоприятным вариантом течения заболевания.

Таким образом, рецидив ВС мы наблюдали значимо чаще среди пациенток, которым в послеоперационном периоде противоспаечный барьер не вводился, а также у женщин с исходно тяжелыми формами заболевания. Этот факт необходимо учитывать при проведении профилактических и реабилитационных мероприятий пациенткам с данной патологией, пролонгируя частоту внутриматочного введения противоспаечного барьера.

Следует отметить, что при морфологическом исследовании биоптата эндометрия, взятого во время контроль-

\section{Таблица 3. Степень тяжести ВС у женщин обследуемых групп (по классификации ESH)}

\begin{tabular}{|c|c|c|c|}
\hline $\begin{array}{c}\text { Степень } \\
\text { тяжести } \\
\text { BC }\end{array}$ & $\begin{array}{c}\text { I группа } \\
\text { обследуемых } \\
(n=48) \text { Абс., \% }\end{array}$ & $\begin{array}{c}\text { II группа } \\
\text { обследуемых } \\
(n=16) \text { Абс., \% }\end{array}$ & $\begin{array}{c}\text { Достоверность } \\
\text { различий, p }\end{array}$ \\
\hline 1 & $1(2,1 \%)$ & $2(12,5 \%)$ & $p>0,05$ \\
\hline II & $22(45,8 \%)$ & $5(31,3 \%)$ & $p>0,05$ \\
\hline III & $19(39,6 \%)$ & $7(43,8 \%)$ & $p>0,05$ \\
\hline IV & $5(10,4 \%)$ & $2(12,5 \%)$ & $p>0,05$ \\
\hline $\mathrm{Va}$ & $1(2,1 \%)$ & - & $p>0,05$ \\
\hline
\end{tabular}


Таблица 4. Количество и частота введения противоспаечного геля Антиадгезин ${ }^{\circledR}$ у пациенток I группы с различной степенью ВC

\begin{tabular}{|c|c|l|}
\hline $\begin{array}{c}\text { Grenень BC } \\
\text { (по ЕSH) }\end{array}$ & $\begin{array}{c}\text { Количество } \\
\text { введений }\end{array}$ & Частота введений \\
\hline I & № 1 & Сразу после операции \\
\hline II & № 2 & $\begin{array}{l}\text { Сразу после операции } \\
\text { и через 5 дней после нее }\end{array}$ \\
\hline III & № 3 & $\begin{array}{l}\text { Сразу после операции } \\
\text { и каждые 4-5 дней после нее }\end{array}$ \\
\hline IV & № 4 & $\begin{array}{l}\text { Сразу после операции } \\
\text { и каждые 3-4 дня после нее }\end{array}$ \\
\hline Va & № 4 & $\begin{array}{l}\text { Сразу после операции } \\
\text { и каждые 3-4 дня после нее }\end{array}$ \\
\hline
\end{tabular}

Таблица 5. Исходы репродуктивной функции у больных обследуемых групп с BC

\begin{tabular}{|l|c|c|c|}
\hline & $\begin{array}{c}\text { I группа } \\
\text { обследуемых } \\
(\mathrm{n}=48) \text { Абс., \% }\end{array}$ & $\begin{array}{c}\text { II пруппа } \\
\text { обследуемых } \\
(\mathrm{n}=16) \text { Абс., \% }\end{array}$ & $\begin{array}{c}\text { Достоверность } \\
\text { различий, p }\end{array}$ \\
\hline Беременность & $16(33,3 \%)$ & $3(18,8 \%)$ & $\mathrm{p}=0,041$ \\
\hline Роды & $5(10,4 \%)$ & $1(6,3 \%)$ & $\mathrm{p}>0,05$ \\
\hline $\begin{array}{l}\text { Неразвивающаяся } \\
\text { беременность }\end{array}$ & $3(6,3 \%)$ & $1(6,3 \%)$ & $\mathrm{p}>0,05$ \\
\hline $\begin{array}{l}\text { Самопроизвольный } \\
\text { выкидыш }\end{array}$ & $3(6,3 \%)$ & $1(6,3 \%)$ & $\mathrm{p}>0,05$ \\
\hline $\begin{array}{l}\text { Прогрессирующая } \\
\text { беременность }\end{array}$ & $5(10,4 \%)$ & - & $\mathrm{p}>0,05$ \\
\hline
\end{tabular}

ной офисной гистероскопии, в 100\% случаев были вновь описаны признаки хронического эндометрита. Это еще раз свидетельствует о наличии серьезных морфологических нарушений в структуре слизистой матки у больных с BC и диктует необходимость проведения в послеоперационном периоде комплекса реабилитационных мероприятий продолжительностью не менее 2-3 менструальных циклов. С этой целью могут применяться: циклическая гормональная терапия, средства, улучшающие кровообращение и стимулирующие ангиогенез. При инфекционной этиологии ВС выполняется бактериологическое исследование мазков и материалов вакуумной биопсии, проводится антибактериальная терапия. Кроме того, в целях успешной регенерации эндометрия в литературе описан опыт применения физиопроцедур, орошения полости матки растворами, кавитированными низкочастотным ультразвуком, гидролизата плаценты человека, тромбоцитарного колониестимулирующего фактора роста, эндометриальных мезенхимальных стволовых клеток [28-31].

Все наблюдаемые нами больные в послеоперационном периоде в течение 2 мес. получали комплексную реабилитационную терапию, включавшую антибиотики (по показаниям), циклическую гормонотерапию, физиолечение. Как видно из данных, представленных в таблице 5, у женщин I группы беременность наступила значимо чаще ( $p=0,041)$ - у каждой третьей больной. При этом у 7 пациенток беременность была спонтанной, в 8 случаях наступила в результате ЭКО. В данный момент 8 обследуемым I группы проводятся реабилитационные мероприятия в послеоперационном периоде, 4 женщины вступили в циклы ЭКО.

Таким образом, для комплексного лечения пациенток с BC решающее значение имеют не только бережное и полное рассечение спаек, но и активный послеоперационный реабилитационный период, включающий профилактику рецидива формирования спаек (за счет проведения при необходимости антибактериальной или противовирусной терапии, а также внутриматочного введения противоспаечных барьеров), мероприятия, направленные на восстановление рецептивности эндометрия и его полноценной функции. Применение противоспаечного геля на основе ГК и карбоксиметилцеллюлозы в комплексе с гормональным лечением является современным инновационным методом профилактики внутриматочного спайкообразования с высоким процентом успешности. При этом необходимо учитывать тяжесть адгезивного процесса в полости матки. Мы считаем целесообразным включение в комплекс послеоперационных реабилитационных мероприятий повторное внутриматочное введение противоспаечного барьера Антиадгезин ${ }^{\circledR}$ пациенткам с тяжелой степенью ВС. При этом частота и кратность его применения должны быть индивидуальными - в зависимости от особенностей анамнеза, морфологической структуры биоптата эндометрия, а также степени спаечного процесса.

Будущие исследования должны быть направлены на изучение клеточных и молекулярных аспектов регенерации эндометрия, а также на разработку мер профилактики первичных и повторных послеоперационных ВС [9].

Конфликт интересов: авторы заявляют об отсутствии конфликта интересов в ходе написания данной статьи.

\section{ЛИТЕРАТУРА}

1. Asherman J.G. Traumatic intra-uterine adhesions. The Journal of Obstetrics and Gynaecology of the British Empire, 1950, 57(6): 892-896.

2. Panayiotides I, Weyers S, Bosteels J, Van Herendae B. Intrauterine adhesion (IUA): Has there been progress in understanding and treatment over last 20 years? Gynecol Surg, 2009, 6: 197-211.

3. March CM. Managemen to Asherman's syndrome. Reprod Biomed Online, 2011, 23 : 63-76.

4. Yu D, Wong YM, Cheong Y, Xia E, Li TC. Asherman syndrome-one century later. Fertil Steril, 2008, 89: 759-779.
5. Panayiotides I, Weyers S, Bosteels J, Van Herendae B. Intrauterine adhesion (IUA): Has there been progress in understanding and treatment over last 20 years? Gynecol Surg, 2009, 6: 197-211.

6. Van Herendael BJ. Treatment of intra-uterine adhesions. Invited presentations and presentations by organisations and societies. 
International Journal of Gynecology \& Obstetrics, 2012, 119S3: 161-260.

7. Yu D, Wong YM, Cheong Y, Xia E, Li TC: Asherman syndrome-one century later. Fertil Steril, 2008, 89: 759-779.

8. Новикова П.В. Возможности использования стволовых клеток различного происхождения в терапии синдрома Ашермана в эксперименте. Журнал акушерства и женских болезней, Спецвыпуск, 2017, LXVI, Национальный конгресс «Дискуссионные вопросы современного акушерства» 15-17 июня 2017 г.: 132-133. /Novikova PV. Therapeutic potential of stem cells of various origins in Asherman syndrome therapy in the experiment. Zhurnal Akusherstva i Zhenskikh Bolezney, Special Issue, 2017, LXVI, National Congress Discussion Issues of Modern Obstetrics, June 15-17, 2017: 132-133.

9. Попов А.А., Мананникова Т.Н., Алиева А.С., Федоров А.А., Беспалова А.Г. Внутриматочные синехии: век спустя. РМЖ, 2017, 12: 895-899. Popov AA, Mannnikova TN, Aliyeva AS, Fedorov AA, Bespalova AG. Intrauterine sinechia: a century later. RMJ, 2017, 12: 895-899.

10. Давыдов А.И., Стрижаков А.Н., Новрузова Н.Х Осложнения оперативной гистероскопии: профилактика и лечение. Вопросы гинекологии, акушерства и перинатологии, 2016, 15(6): 52-60. /Davydov Al, Strizhakov AN, Novruzova NKh. Complications of operative hysteroscopy: prevention and treatment. Voprosy Ginekologii, Akusherstva i Perinatologii, 2016, 15 (6): 52-60.

11. Takai IU, Kwayabura AS, Ugwa EA, Idrissa A, Obed JY, Bukar MA. 10-year review of the clinical presentation and treatment outcome of Asherman's syndrome at a center with limited resources. Ann Med Health Sci Res, 2015, 5(6): 442-446.

12. Ibrahim MI, Raafat TA, Ellaithy MI, Aly RT. Risk of postpartum uterine synechiae following uterine compression suturing during postpartum haem- orrhage. Aust N ZJ ObstetGynaecol, 2013, 53(1): 37-45.

13. Renier D, Bellato $P$, Bellini $D$ et al. Pharmacokinetic behavior of ACP gel, an autocrosslinkedhyaluronan derivative, after intraperitoneal administration. Biomaterials, 2005, 26(26): 53-68

14. Pellicano M, Guida M, Zullo F et al. Carbon diox ide versus normal saline as a uterine distension medium for diagnostic vaginiscopie hysteroscopy in infertile patients: a prospective, randomized, multicenter study. FertilSteril, 2003, 79: 418-421.

15. Stefanescu A, Marinescu B. Diagnostic hysteroscopy - a retrospective study of 1545 cases. Maedica (Buchar), 2012, 7(4): 309-314.

16. Conforti A, Alviggi C, Mollo A, De Placido G, Magos A. The management of Asherman syndrome: a review of literature. Reprod Biol Endocrinol, 2013, 27(11): 118.

17. Panayotidis C, Weyers S. Intrauterine adhesions (IUA): has there been progress in understanding and treatment over the last 20 years? Gynecological surgery, 2009, 6(3): 197-211.

18. Jones K. Ambulatory gynaecology: a new concept in the deliveryof healthcare for women. Gynecological Surgery, 2006, 3(3): 153-156.

19. Chinese Society of Obstetrics and Gyneocology Chinese Medical Association. Expert consensus on the diagnosis and management of intrauterine adhesions in China. Zhonghua Fu Chan KeZaZhi, 2015, 50(12): 881-887.

20. March CM. Intrauterine adhesions. Obstet Gynecol Clin North Am, 1995, 22: 491-505.

21. Amer MI, El Nadim A, Hassanein K. The role of intrauterine balloon after operative hysteroscopy in the prevention of intrauterine adhesion:a prospective controlled study. MEFS J, 2005, 10: 125-129.

22. Di SpiezioSardo A., Calagna G., Scognamiglio M., O'Donovan P, Campo R, De Wilde RL. Prevention of intrauterine post-surgical adhesions in hyst- eroscopy. A systematic review. Eur J Obstet Gynecol Reprod Biol, 2016, 203: 182-192.

23. Metwally M, Watson A, Lilford R, Vandekerckhove P: Fluid and pharmacological agents for adhesion prevention after gynaecological surgery. Cochrane Database Syst Rev, 2006, 19: CD001298.

24. Mais V, Cirronis MG, Peiretti M, Ferrucci G, Cossu $\mathrm{E}$, Melis GB. Efficacy of autocrosslinkedhyaluronan gel for adhesion prevention in laparoscopy and hysteroscopy:a systematic review and metaanalysis of randomized controlled trials. Eur J Obstet Gynecol Reprod Biol, 2012, 160: 1-5.

25. The Use of Adhesion Prevention Agents in Obstetrics and Gynaecology, RCOG. Scient Impact Paper, 2013, 39: 6.

26. AAGL Practice Report: Practice Guidelines for Management of Intrauterine Synechiae. 2013: 8.

27. Справочник лекарств РЛС. Антиадгезин гель противоспаечный рассасывающийся стерильный. http://www.rlsnet.ru/pcr_tn id 81752.htm.

28. Wamsteker K, Blok SD. Diagnostic hysteroscopy: technique and docu- mentation. In: Sutton C, Diamond M, editors. Endoscopic Surgery for Gynecologists. Philadelphia, PA: WB Saunders, 1993.

29. Bassil S. Changes in endometrial thickness, width, length and pattern in predicting pregnancy outcome during ovarian stimulation in in vitro fertilization. Ultrasound Obstet Gynecol, 2001, 18(3): 258-263.

30. Preutthipan S, Linasmita V. Reproductive outcome following hystero- scopiclysis of intrauterine adhesions: a result of 65 cases at Ramathibodi Hospital. J Med Assoc Thai, 2000, 83(1): $42-46$.

31. March C, Israel R. Intrauterine adhesions secondary to elective abortion. Hysteroscopic diagnosis and management. Obstet Gynecol, 1976, 48(4): 422-429.

\section{ИННОВАЦИОННАЯ КОНЦЕПЦИЯ ПРОФИЛАКТИКИ СПАЕЧНОЙ БОЛЕЗНИ В ПРАКТИКЕ АКУШЕРА-ГИНЕКОЛОГА}

\section{Противоспаечный гель с уникальным составом' при хирургических операциях в акушерстве и гинекологии}

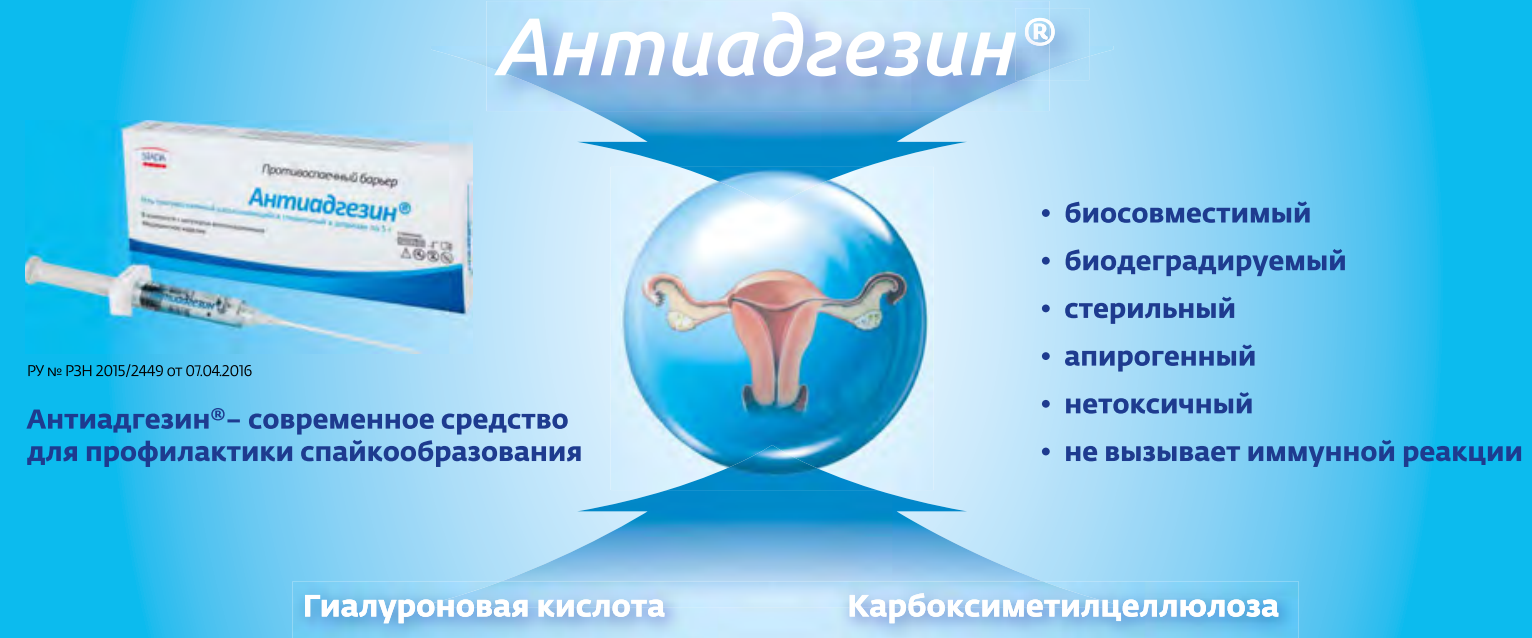

'единственный противоспаечный барьер с данным составом, зарегистрированый в РФ, согтасно базе http://www.roszdravnadzor.ru, декабрь 2015 заказ продукции: АО «Нижфарм», 119017, Москва, ул. Б. Ордынка д. 44, к. 4. Тел./факс (495) 7831303 (доб. 12117), www.stada.ru



